Soyama et al.

\title{
How I do it
}

Tumor thrombectomy via a surgically reopened umbilical vein combined with right hemihepatectomy in a patient with hepatocellular carcinoma

Akihiko Soyama, Susumu Eguchi, Mitsuhisa Takatsuki, Masaaki Hidaka, Izumi Muraoka, Tetsuo Tomonaga, Takashi Kanematsu.

Department of Surgery, Nagasaki University Graduate School of Biomedical Sciences, Nagasaki, Japan

Running head: HCC tumor thrombectomy via umbilical vein Key words: hepatocellular carcinoma; portal venous tumor thrombosis; umbilical vein; occlusion catheter; thrompectomy; right hemihepatectomy.

\section{Address correspondence to:}

Susumu Eguchi, MD, PhD.

Department of Surgery, Nagasaki University Graduate School of Biomedical Sciences, 1-7-1 Sakamoto, Nagasaki 852-8501, Japan

TEL: 81-95-819-7316, FAX: 81-95-819-7319

E-mail: sueguchi@nagasaki-u.ac.jp 
Soyama et al.

\begin{abstract}
This report presents a case of a patient with a huge hepatocellular carcinoma in the right lobe of the liver with extensive portal venous tumor thrombus into the main portal trunk and left portal branch. The patient underwent extended right hemihepatectomy with tumor thrombectomy with direct extraction from an opening of the ventral wall of the right portal vein and using a balloon catheter to push the tumor thrombus via surgically reopened umbilical vein. This technique seems useful in case a tumor thrombus that extended deep into the umbilical portion of the left portal vein. In addition, this technique may have minimized the intraoperative migration of the tumor thrombus into the future remnant liver by occluding portal flow with the balloon catheter during thrombectomy.
\end{abstract}


Soyama et al.

\section{How I do it}

Although portal venous tumor thrombus (PVTT) is regarded as an ominous prognostic factor in patients with hepatocellular carcinoma (HCC), several reports have noted the efficacy of curative resection to achieve an acceptable survival rate. Reported 5-year survival reached around $40 \%$ (1, 2). Right or left hemihepatectomy with segmental resection of the main portal vein has been applied for HCC with PVTT extending into the main portal trunk or the contralateral portal vein, using several modified surgical techniques $(3,4)$. This procedure is considered to be an en bloc resection. Whereas, Inoue et al. reported a comparable outcome with en bloc resection to peel PVTT from the venous wall, a procedure that they call the "peeling off technique" (2). This report describes a case of R0 resection for a patient with a huge HCC in the right liver lobe with PVTT extending into the main portal trunk and left portal branch with thrombectomy using a balloon catheter via the reopened umbilical vein.

A 31 year-old male with upper abdominal pain was referred for HCC in the right liver lobe, which developed in a chronically damaged liver infected with hepatitis virus B. Abdominal computed tomography (CT) scan showed an approximately $12 \mathrm{~cm}$ tumorous lesion in the right lobe with contrast-enhancement in the arterial phase and smooth wash-out of contrast in the delayed phase, which was compatible with HCC. In addition, CT scan showed a PVTT that occupied the right portal vein, and extended into the main portal trunk and left portal vein (Fig. 1). Because of the portal hypertension probably due to the portal venous thrombus, collateral vessels developed around the 
Soyama et al.

hepatic hilum. Endoscopy showed esophageal varices, which did not appear to be risky for rupture (F1, Cb, RC (-)). The laboratory findings were; prothrombin time INR 1.14, serum albumin level $4.2 \mathrm{~g} / \mathrm{dl}$, total serum bilirubin level $0.7 \mathrm{mg} / \mathrm{dl}$, aspartate aminotransaminase 49 IU/L, alanine aminotransferase 55 IU/L, alpha-fetoprotein (AFP) 46,664 ng/ml and protein induced by vitamin $\mathrm{K}$ absence or antagonists-II (PIVKA-II) 5,864 $\mathrm{mAU} / \mathrm{ml}$. The hepatic functional reserve was evaluated with indocyanine green 15min retention rate (ICG-R-15 min) and 99mTc-GSA liver scintigraphy. Hepatic functional reserve was moderately impaired with the ICG-R-15\% of $22 \%$, and the ratio of liver to heart-plus-liver radioactivity of Tc-GSA 15 minutes after injection (LHL15) was 0.877 in $99 \mathrm{mTc}$-GSA liver scintigraphy. No other image examinations revealed extrahepatic lesions. These findings indicated that extended right hemihepatectomy with tumor thrombectomy was the optimal procedure for curative resection. The LHL15 values of 0.90 in $99 \mathrm{mTc}-\mathrm{GSA}$ liver scintigraphy indicated the type of hepatectomy. Although the hepatic functional reserve was not considered ideal for a major hepatectomy, the liver synthetic function was normal. The patient was young and physically healthy, apart from the hepatic lesion. The patient underwent extended right hemihepatectomy with tumor thrombectomy as planned, after providing informed consent with a detailed explanation of the peri-operative complications including possible post-operative liver failure.

The ventral wall of the right portal branch was opened with adequate vascular control of portal flow for thrombectomy. The main trunk and right branch of the portal vein was clamped. Subsequently, the tumor thrombus was extracted via the opening 
Soyama et al.

with occasional flushing of portal blood flow. It was not possible to achieve complete thrombectomy only via the opening of the right portal vein. Although a Fogarty catheter was introduced via the opening to pull the tumor thrombus to proximal side of the portal vein, thrombectomy was markedly difficult because there was insufficient space to inflate the balloon because the umbilical portion was almost completely filled with the tumor thrombus (Fig. 2). Therefore, an occlusion catheter (9Fr, 18mm) was introduced from a surgically reopened umbilical vein to achieve complete thrombectomy by pushing the inflated balloon from the distal end of the tumor thrombus in the umbilical portion of the portal vein toward the main portal trunk (Fig. 3). As a result, tumor thrombus was completely removed via the opened the right portal vein by pushing the occlusion catheter through the left portal vein. Intraoperative US did not show any remnant thrombus, and subsequent sufficient portal flow was confirmed with Doppler US after the thrombectomy. Liver parenchymal resection we subsequently performed after the thrombectomy, to achieve extended right hemihepatectomy. The patient was doing well 6 months after the surgery. Follow-up abdominal CT scan showed a patent portal venous system with no evidence of a recurrent tumor or tumor thrombus.

The "peeling off technique" is relatively simple compared to en-bloc resection because it does not require the resection and reconstruction of the portal vein. Inoue et al. reported a longitudinal incision of umbilical portion of the left portal vein as a useful technique to perform tumor thrombectomy with the "peeling off technique" in that area (2). Indeed, it is a good option especially in a case with a left portal system dominant tumor thrombus. However, there is a risk of portal venous stenosis after the closure of 
Soyama et al.

the longitudinal incision with this technique. The left liver lobe should be reserved after a curative right hemihepatectomy in patients with portal venous tumor thrombus that originates from a tumor in the right lobe. The current technique avoids the reconstruction of the portal vein and maintains a good portal flow to the remnant liver.

Thrombectomy with a Fogarty catheter can be markedly difficult when the portal venous tumor thrombus extends to the entrance of the umbilical vein; because there is frequently insufficient space is to inflate the balloon to pull the tumor thrombus to the proximal side of the portal vein. A catheter was inserted via reopened umbilical vein to overcome this problem in the current case. In addition, the use of an occlusion catheter might be able to minimize the intraoperative migration of the tumor thrombus into the future remnant liver.

Hepatectomy with tumor thrombectomy using an occlusion catheter via the umbilical vein for HCC in the right lobe with PVTT extended into left portal vein seems safe and reasonable, not only from a technical point of view, but also from an oncological point of view. 
Soyama et al.

\section{References}

1. Fukuda S, Okuda K, Imamura M, Imamura I, Eriguchi N, Aoyagi S. Surgical resection combined with chemotherapy for advanced hepatocellular carcinoma with tumor thrombus: report of 19 cases. Surgery. 2002;131:300-10.

2. Inoue Y, Hasegawa K, Ishizawa T, Aoki T, Sano K, Beck Y, et al. Is there any difference in survival according to the portal tumor thrombectomy method in patients with hepatocellular carcinoma? Surgery. 2009;145:9-19.

3. Wu CC, Hsieh SR, Chen JT, Ho WL, Lin MC, Yeh DC, et al. An appraisal of liver and portal vein resection for hepatocellular carcinoma with tumor thrombi extending to portal bifurcation. Arch Surg. 2000;135:1273-9.

4. Yamaoka Y, Kumada K, Ino K, Takayasu T, Shimahara Y, Mori K, Tanaka A, Morimoto T, Taki Y, Washida M, et al. Liver resection for hepatocellular carcinoma (HCC) with direct removal of tumor thrombi in the main portal vein. World J Surg. 1992;16:1172-6. 
Soyama et al.

Figure legends

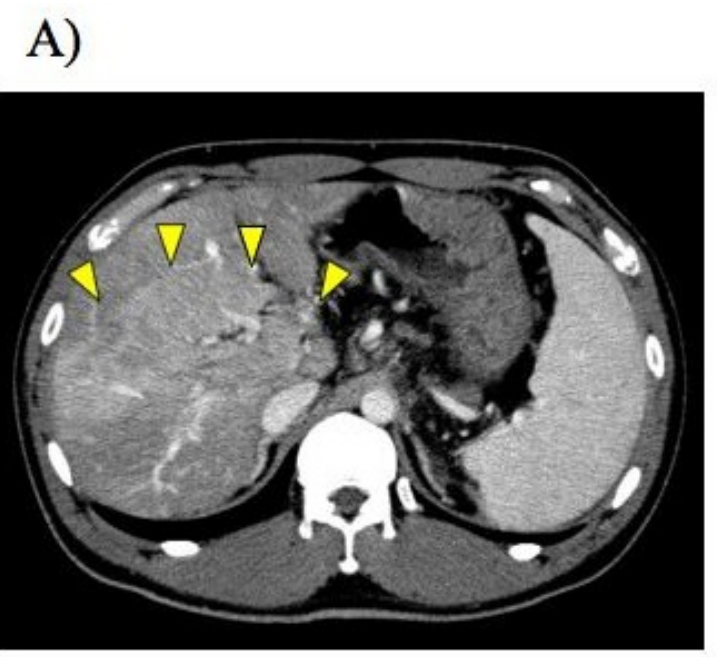

B)

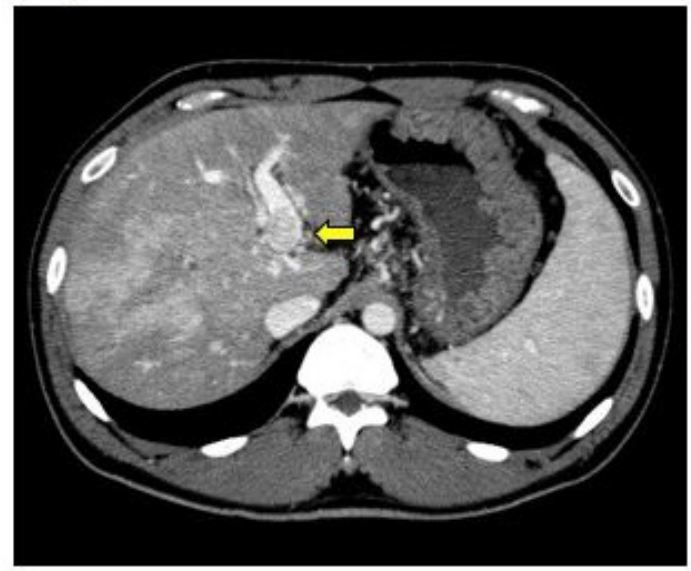

Figure 1. A) Contrast enhanced computed tomography showed huge tumor in the right lobe of the liver with portal venous tumor thrombus (PVTT) (arrowheads). B) PVTT extended into the umbilical portion of the left portal vein (arrow). 
Soyama et al.

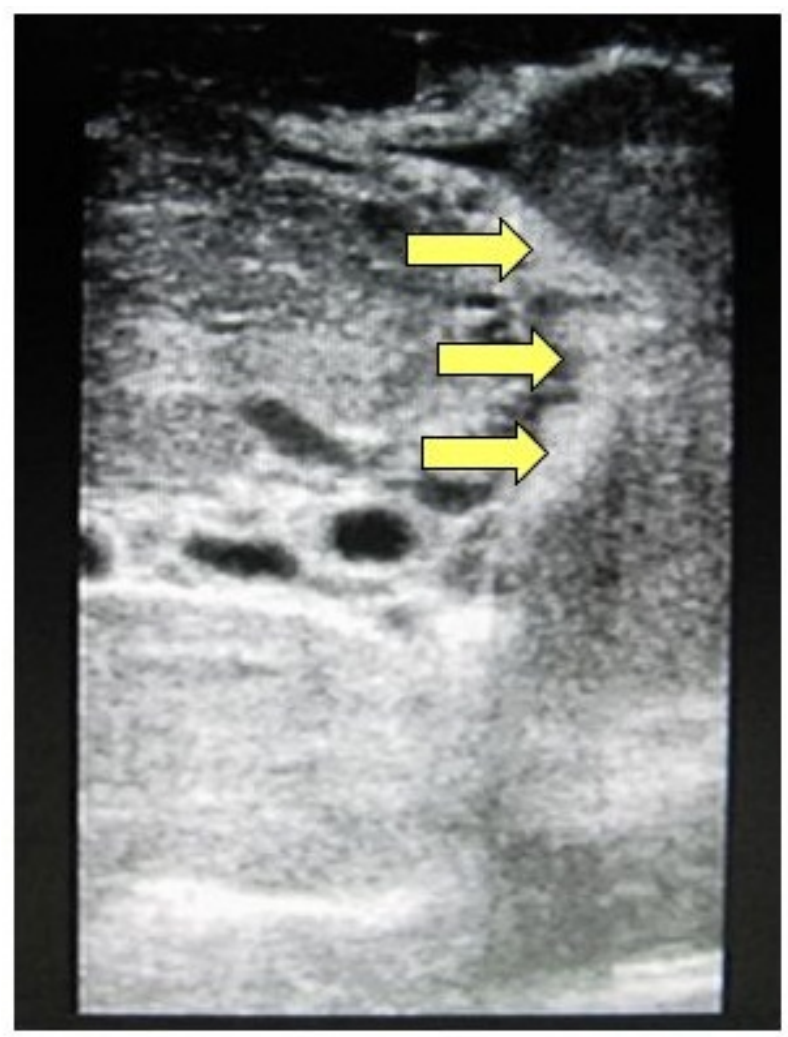

Figure 2. Intraoperative ultrasound showed the tumor thrombus in the umbilical potion of the left portal vein that had more extended in comparison to the preoperative computed tomography. 


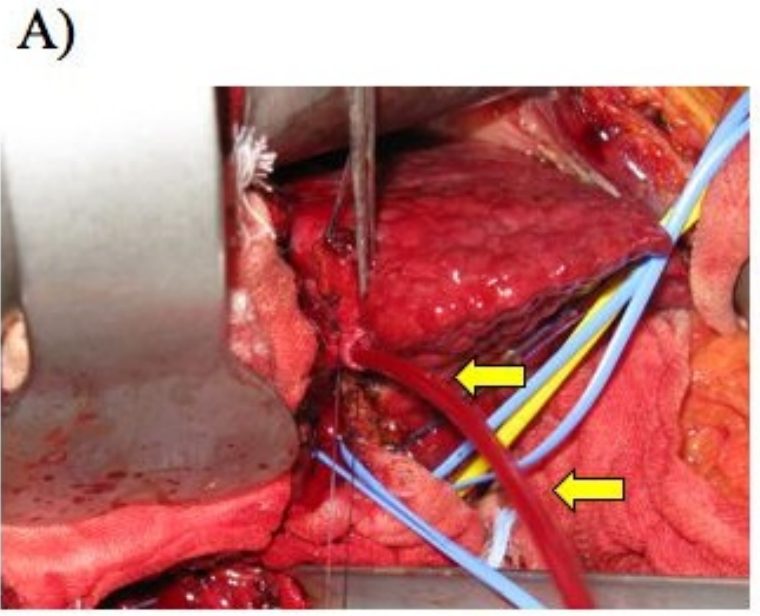

\section{B)}

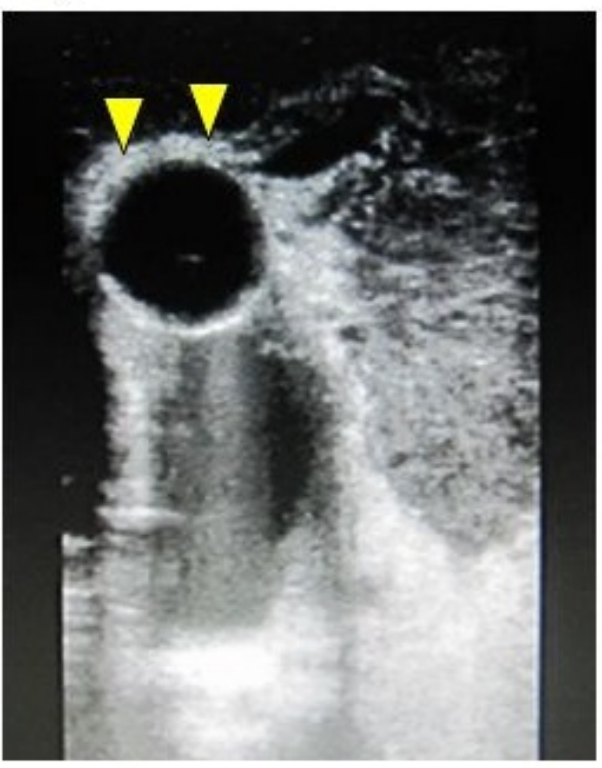

C)

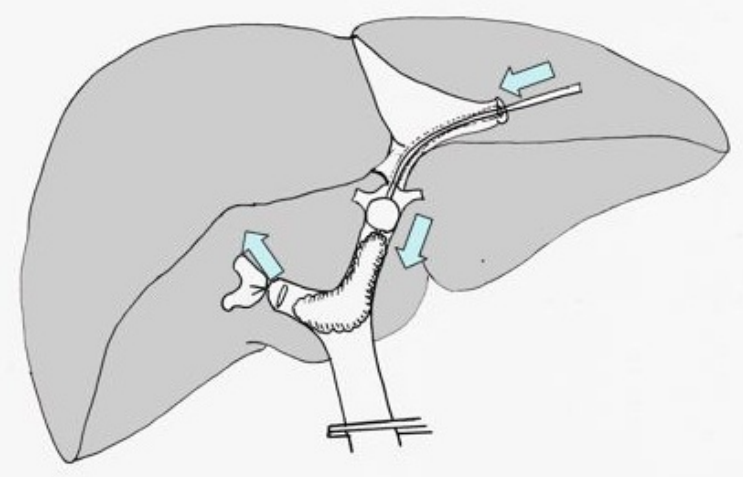

Figure 3. A) Surgical view. A balloon catheter (arrows) was introduced via the surgically reopened umbilical vein. B) Arrow heads show the balloon of the catheter was inflated (arrowheads) within the umbilical portion of the left portal vein. C) Schematic diagram of the surgical procedure. A balloon catheter was pushed through the reopened umbilical vein towards the opening of the right portal vein. 